\title{
Educação nutricional para adultos e idosos: uma experiência positiva em Universidade Aberta para a Terceira Idade ${ }^{1}$
}

\author{
Nutrition education for adults and the elderly: \\ an experience in Open University for the Third Age
}

Ana Maria CERVATO 2

Alice Moreira DERNTL2

Maria do Rosário Dias de Oliveira LATORRE²

Maria de Fátima Nunes MARUCCI²

\section{RES U M O}

\section{Objetivo}

Avaliar uma intervenção nutricional educativa desenvolvida para alunos de Universidades Abertas para a Terceira Idade.

\section{Métodos}

A população foi constituída por indivíduos com 45 anos de idade ou mais, freqüentadores de quatro instituições de ensino do Município de São Paulo. O estudo teve delineamento quase experimental, do tipo pré-teste/ pós-teste, sem grupo controle. A ação educativa (quatro aulas de três horas cada; distribuição de uma apostila com o conteúdo das aulas e de um guia alimentar com orientações gerais) era parte das atividades oferecidas pelas instituições e foi desenvolvida por nutricionistas durante o segundo semestre de 1996. Os dados pessoais e de conhecimentos sobre nutrição foram coletados por meio de questionário auto-aplicado. 0 conhecimento foi identificado aplicando-se teste especialmente elaborado. As práticas alimentares, identificadas por meio do registro de alimentos consumidos em três dias, tiveram como variáveis analisadas: o valor energético total, a proporção de macronutrientes, o colesterol, a vitamina A, cálcio e ferro.

\section{Resultados}

As modificações identificadas foram: aumento dos conhecimentos sobre nutrição, diminuição de consumo de lipídios, de proteínas e de colesterol. As modificações citadas referiram-se ao tipo de alimento consumido, à

\footnotetext{
1 Artigo elaborado a partir da tese de A.M.CERVATO, "Intervenção nutricional educativa: promovendo a saúde de adultos e idosos em Universidades Abertas para Terceira Idade”. Faculdade de Saúde Pública, Universidade de São Paulo, 1999. 227p.

2 Faculdade de Saúde Pública, Universidade de São Paulo. Av. Dr Arnaldo, 725, 01204-906, São Paulo, SP, Brasil. Correspondência para/Correspondence to: A. M. CERVATO.E-mail: <cervato@usp.br>.
} 
ingestão de água e à maneira de preparar os alimentos. Estas modificações ocorreram por motivos de saúde, e as fontes de informações sobre nutrição mais citadas, foram a Universidade Aberta para a Terceira Idade e o médico pessoal.

\section{Conclusão}

Apesar das limitações do estudo, os resultados indicaram que, em função dessa atividade, houve uma tendência para a modificação da dieta e dos conhecimentos sobre nutrição.

Termos de indexação: dietas, educação nutricional, idoso, saúde pública, saúde do adulto, serviços de saúde para idosos.

\section{A B S T R A C T}

\section{Objective}

To evaluate an educational nutrition intervention developed for students of the Open Universities for the Third Age.

\section{Methods}

The studied population was constituted by 44 individuals, 45 years of age or older, who attend four teaching institutions in São Paulo County. The general plan consisted of a quasi-experimental study of the kind pre/test and post/test verifications, with no control group. The educational actions consisted of four three-hour classes, and the distribution of a study outline with the classes' contents and a food-guide with general information. Such actions were included as part of the activities offered by the institutions and were developed by nutritionists during the second term of 1996. The personal data and knowledge about nutrition were gathered through a self-applied questionnaire. The knowledge was verified applying specially elaborated test. The alimentary practices, verified through a three-day food diary, had the following variables analyzed: total energetic value, macronutrient proportion, cholesterol, vitamin A, calcium and iron content.

\section{Results}

For this group of individuals, the identified modifications were: the increased knowledge about nutrition and a decreased consumption of lipids, proteins and cholesterol. Such modifications referred to the kind of food consumed, amount of water ingestion, and ways of preparing foods. These alterations were motivated by for health reasons, and the most mentioned information sources were the Open University and the personal physician

\section{Conclusion}

On account of the educational activity, and in spite of the study limitations, the results showed a positive trend in diet modification and increased knowledge about nutrition.

Indexing terms: diet, nutrition education, aged, adult health, public health, health services for the aged.

\section{N T R O D U Ç Ã O}

No Brasil, e em diversos outros países em desenvolvimento, o aumento da população idosa vem ocorrendo de forma muito rápida, sem a correspondente modificação nas condições de vida. Problemas de saúde, como doenças infecto-contagiosas e parasitárias, ainda persistem sem solução, bem como as doenças crônicas não transmissíveis, cuja incidência aumenta, ocupando lugar de destaque no perfil de mortalidade desses países. Esse envelhecimento populacional 
determina um substancial aumento, tanto nos recursos materiais e humanos necessários aos serviços de saúde do país, como nos seus custos, visto que, em geral, as doenças que acometem essa camada da população necessitam de tratamento por períodos prolongados e intervenções caras com alta tecnologia ${ }^{1}$.

As investigações vêm mostrando formas de melhorar a qualidade de vida daqueles que estão no processo de envelhecer, e uma das formas de se obter essa melhoria é o desenvolvimento de programas de saúde. Segundo a Organização Mundial da Saúde (OMS), esses programas de saúde, que têm como base a comunidade e que implicam promoção da saúde do idoso, devem ter a nutrição como uma das áreas prioritárias².

No processo de envelhecimento a importância da alimentação é comprovada por estudos epidemiológicos, clínicos e de intervenção, entre outros, que têm demonstrado ligação consistente entre o tipo de dieta e o surgimento de doenças crônicas não transmissíveis, incluindo as doenças cardíacas coronarianas, doenças cérebro-vasculares, vários tipos de cânceres, diabetes melito, cálculos biliares, cáries dentárias, distúrbios gastrointestinais e várias doenças ósseas e de articulações ${ }^{3}$.

A educação nutricional tem sido destaque de distintos trabalhos epidemiológicos, em especial aqueles nos quais os resultados apontam para a correlação entre comportamento alimentar e doenças ${ }^{3,4}$.

Aranceta-Bartrina ${ }^{5}$ conceitua educação nutricional como "parte da nutrição aplicada que orienta seus recursos em direção à aprendizagem, adequação e aceitação de hábitos alimentares saudáveis". Tal conceito, segunda a autora, está em consonância com os conhecimentos científicos em nutrição e persegue o objetivo último de promoção de saúde do indivíduo e da comunidade. Contento et al. ${ }^{6}$ definem a educação nutricional como qualquer experiência de ensino desenvolvida para facilitar a adoção voluntária de comportamento alimentar ou outro relacionado à nutrição, com a finalidade de conduzir à situação de saúde e bem-estar.

Desenvolvida desde uma perspectiva problematizadora ou participativa, a educação nutricional é um estímulo à transformação do educando. Ele passa de uma situação na qual sua conduta alimentar é determinada pelo condicionamento e pelo hábito repetido mecanicamente, para outra, na qual ele, compreendendo seu próprio corpo e aprendendo a ouvi-lo e observá-lo, passa a se tornar sujeito de sua conduta alimentar?.

Um conceito educativo que vem sendo adotado nos últimos anos, em especial com a população idosa, refere-se ao auto-cuidado. Compreende todas as ações e decisões que uma pessoa realiza para prevenir, diagnosticar e tratar uma enfermidade, todas as atividades individuais destinadas a manter e melhorar a saúde e, ainda, as decisões de utilizar tanto os sistemas de apoio formal de saúde quanto os informais ${ }^{8}$. Está, portanto, intimamente ligado à educação para a saúde ${ }^{9}$. O auto-cuidado é a ação realizada por uma pessoa adulta a fim de se cuidar ${ }^{9}$. O indivíduo, comprometido com esse processo, prepara-se e capacita-se, deliberadamente, para atuar sobre os fatores que afetam seu funcionamento e desenvolvimento. A educação nutricional é uma ferramenta que dá autonomia ao educando, para que ele possa assumir, com plena consciência, a responsabilidade pelos seus atos relacionados à alimentação ${ }^{7}$ e está em consonância com a estratégia educativa do auto-cuidado.

A opção pelo auto-cuidado como estratégia educativa congrega atividades para a promoção da saúde, para a modificação do estilo de vida prejudicial à saúde, para a diminuição dos fatores de risco e prevenção específica de doenças, para a manutenção e recuperação da saúde e, por fim, para a reabilitação 9 . Nesta opção educativa, o profissional de saúde, em função de sua habilitação e responsabilidade, desempenha o papel de facilitador do processo de mudança. Toda e qualquer intervenção nutricional educativa 
terá maiores chances de sucesso, se estiver incluída em programas habituais que promovam pequenas e confortáveis, mas importantes mudanças, por um longo período de tempo ${ }^{10}$.

Dentro desta perspectiva de atividades habituais, estão inseridos os cursos para a população adulta e idosa, sob a denominação de Universidade Aberta para a Terceira Idade (UATI). O objetivo destes cursos é atender a uma das necessidades da população idosa, que é a demanda educacional, por meio de várias propostas e recursos educativos ${ }^{11}$.

A partir de 1990, várias instituições paulistas de ensino tomaram a iniciativa de desenvolver cursos e atividades específicas para indivíduos com idade superior a 45 anos, com o objetivo de desenvolvimento e enriquecimento pessoal11,12. Apesar das mais diversas atividades disponíveis, em geral, a estrutura dos cursos oferecidos pelas instituições brasileiras de ensino, principalmente pelas instituições paulistas, é muito semelhante. Tal estrutura baseia-se na proposta desenvolvida pela Pontifícia Universidade Católica de São Paulo (PUC-SP) ${ }^{11}$, em que constam três módulos com duração de um semestre cada um, com duas aulas semanais de três horas cada uma. Enfocando diversos temas, as atividades abordadas nos módulos são: reciclagem e atualização cultural, em que são ministradas disciplinas como Sociologia, Antropologia, Política, Filosofia, Direito e outras; orientações práticas para uma vida saudável, em que há atuação de especialistas nas áreas de: Geriatria, Gerontologia, Nutrição, Fonoaudiologia e outras; atividades socioculturais e educativas, com o envolvimento do corpo discente em atividades que incentivam sua sociabilidade e seu interesse pela ação e produção intelectual ${ }^{11}$.

O papel das UATI tem sido discutido em diferentes trabalhos. A maioria deles ressalta a importância destes projetos ligados às instituições de ensino, tanto como atividade de extensão, quanto de pesquisa e ensino.

Em pesquisa com freqüentadores das atividades realizadas por uma instituição de ensino no interior do estado de São Paulo, verificou que os temas relacionados à saúde são aqueles de maior aceitação ${ }^{12}$.

Por meio de depoimentos, o projeto de promoção da saúde da Universidade Aberta da Terceira Idade, da Universidade Estadual do Rio de Janeiro, foi avaliado pelos freqüentadores como um espaço de aprendizagem e apoio social com repercussões positivas na auto estima e na postura frente à vida e ao envelhecimento ${ }^{14}$.

Considerando as diferentes iniciativas de implantação e desenvolvimento de atividades com a população adulta e idosa, em várias Universidades e/ou Faculdades, e a preocupação dos dirigentes desses cursos em estabelecer a "Nutrição" como um dos temas a serem abordados, a presente pesquisa teve por objetivo avaliar uma intervenção nutricional educativa, desenvolvida junto à população freqüentadora de quatro instituições da rede particular de ensino superior, localizadas no município de São Paulo.

\section{CASUÍSTICA E MÉTODOS}

A população de estudo foi composta por 44 alunos de ambos os sexos, matriculados no primeiro módulo de projetos denominados "Universidades Abertas para a Terceira Idade", vinculados a quatro instituições de ensino, localizadas no município de São Paulo: Instituto de Educação Costa Braga (zona sul do Município), Pontifícia Universidade Católica (zona oeste), Faculdades Santana (zona norte) e Universidade São Judas Tadeu (zona leste).

O critério para escolha dessas instituições decorreu da autorização da coordenação dos projetos para a realização e avaliação da intervenção nutricional educativa, como parte das atividades oferecidas aos alunos matriculados.

A intervenção nutricional educativa se refere ao conjunto de atividades desenvolvidas por um grupo de nutricionistas, previamente treinados, envolvidos com os projetos Universidades Abertas para a Terceira Idade (UATI) das instituições 
anteriormente citadas. Essa intervenção foi desenvolvida no segundo semestre de 1996, entre os meses de agosto e novembro.

A avaliação da intervenção teve caráter quase experimental, do tipo pré-teste/pós-teste, no qual o indivíduo é o seu próprio controle. Para tanto, os alunos foram convidados a colaborar, preenchendo os questionários elaborados para conhecer o consumo alimentar e as noções conceituais sobre nutrição, referentes aos 20 dias anteriores e aos seis meses posteriores à intervenção. A participação neste preenchimento foi voluntária, não vinculada à participação na intervenção.

Os instrumentos de pesquisa foram questionários auto-aplicados, contendo questões semi-estruturadas, escolhidas em função dos objetivos, da população de estudo e da viabilidade da coleta dos dados. Foram utilizados dois questionários: um, aplicado na fase pré-teste, e outro, na fase pós-teste.

No questionário do pré-teste incluíram-se as perguntas sobre dados pessoais, o teste de noções conceituais sobre nutrição e a ficha para registro do consumo alimentar. A distribuição destes questionários foi realizada de acordo com o cronograma das instituições, sob criteriosa orientação para o seu preenchimento correto. Após uma semana, foram devolvidos para verificação da qualidade das anotações e, quando necessário, solicitou-se maior detalhamento do conteúdo.

A outra avaliação ocorreu baseada nos resultados do pós-teste, realizado seis meses após a primeira intervenção. O pós-teste constituiu-se de um questionário auto-aplicado, incluindo o teste de noções sobre nutrição, a ficha de registro de alimentos consumidos e os dados referentes às mudanças no comportamento alimentar, ocorridas no último ano. Esse questionário foi distribuído pela própria autora da pesquisa, visando minimizar a influência que o relacionamento aluno-professor poderia ter nas respostas.

O teste de noções sobre nutrição compreendeu um conjunto de temas, cujo conteúdo foi transformado em perguntas. As perguntas foram elaboradas com base na literatura e, principalmente, nas dúvidas apresentadas pelos alunos durante o desenvolvimento das atividades nas Universidades Abertas da Terceira Idade.

A primeira parte das perguntas correspondeu às noções sobre composição química dos alimentos, ou seja, o seu valor nutritivo. Foram elaboradas quatro perguntas referentes aos alimentos ricos em colesterol, aos alimentos equivalentes, às presenças de substâncias diet $\mathrm{e}$ de nutrientes antioxidantes.

A segunda parte questionou a função dos nutrientes no organismo. Foram elaboradas cinco perguntas sobre os alimentos energéticos, os alimentos reguladores, a função das fibras, das gorduras e do cálcio, juntamente com a vitamina D.

A terceira parte foi composta por quatro outras perguntas referentes às características de uma dieta equilibrada, tanto a de uma forma geral, como a de uma forma específica para indivíduos idosos.

O consumo alimentar foi identificado com o registro alimentar de três dias nas fichas distribuídas com os questionários. Essa ficha abrangeu três dias intercalados, sendo, preferencialmente, mas não obrigatório, que pelo menos um destes fosse referente ao fim de semana (sábado ou domingo).

As informações obtidas em medidas caseiras foram convertidas em gramas de alimentos crus, tomando como referência os valores das medidas caseiras encontrados na literatura e calculados com base no Programa de Apoio à Decisão em Nutrição da Universidade Federal de São Paulo' ${ }^{15}$, adaptado aos objetivos da presente pesquisa.

A avaliação da intervenção educativa baseou-se nas mudanças ocorridas nas seguintes variáveis de consumo alimentar: valor energético total (expresso em kcal); macronutrientes (expressos em porcentagem de energia fornecida por proteínas, lipídios e carboidratos); colesterol 
(expresso em mg); cálcio (expresso em mg); vitamina $A$ (expressa em equivalente retinol em $\mathrm{mg}$ ) e Ferro (expresso em mg).

As noções conceituais foram avaliadas, tanto de modo específico, como global. A avaliação específica significou as alterações de acerto em cada pergunta. Cada uma das 13 questões tinha peso $=1$ e apresentava uma ou mais alternativas certas. O número destas variou de 1 a 13, dependendo da pergunta. Dessa forma, o peso 1 da questão foi dividido proporcionalmente, em cada pergunta, entre as alternativas certas. Entretanto, ao assinalar alternativas erradas, independentemente de ter assinalado outras certas, ou escolher a alternativa "não sei", a pontuação da pergunta foi "zero". A variabilidade da pontuação foi entre "zero" e "13", em decorrência do número de questões.

A avaliação global do teste de noções conceituais sobre nutrição, foi determinada pela somatória da proporção de acerto às perguntas formuladas, indicando a pontuação de cada indivíduo.

Além dessas variáveis, outras foram avaliadas e identificadas a partir de questões específicas sobre as modificações realizadas nas dietas e sobre as fontes de informação importantes na determinação e/ou colaboração para as mudanças na dieta. Os dados, depois de coletados, foram armazenados e analisados, utilizando-se o Programa Epi Info'16.

As mudanças de comportamento foram analisadas tanto do ponto de vista da quantidade de energia da dieta e de nutrientes consumidos, quanto das noções conceituais sobre nutrição. Foi analisada, para cada variável dietética (energia total; contribuição energética dos carboidratos, dos lipídios e das proteínas; quantidade total de colesterol, de cálcio, de ferro e de vitamina A) a diferença entre a média do valor inicial (antes da intervenção) e a média do valor final (após a intervenção). As noções conceituais foram avaliadas comparando a média da pontuação inicial e a média da pontuação final. Em ambos os casos, foram utilizados o teste " $\mathrm{t}$ ", de Student, para dados pareados. Em todas as análises foi utilizado o nível de significância de 5\%.

A intervenção nutricional educativa constou de duas partes: uma atividade de orientação geral e uma de orientação específica.

A primeira consistiu em aulas de nutrição, ministradas por nutricionistas, que receberam treinamento específico para participarem da pesquisa, integrantes do Grupo de Estudos de Nutrição na Terceira Idade (GENUTI) ${ }^{(3)}$. Tendo em vista que se tratava de uma ação educativa era necessária a opção por uma abordagem que norteasse os processos. Assim, o grupo de professores definiu como diretriz a conscientização dos alunos quanto ao hábito alimentar, mobilizando-os para o auto-cuidado.

A didática utilizada consistiu em aulas interativas, com recursos visuais, um conjunto de transparências e, como apoio didático, uma apostila especialmente elaborada. A carga horária total foi de 12 horas, divididas em 4 aulas, ministradas duas vezes por semana. O conteúdo programático das aulas foi definido pelo grupo de nutricionistas que vinha desenvolvendo esta ação junto à população freqüentadora de cada UATI. Este conteúdo foi definido a partir dos três anos de experiência em aulas - em Projetos de Universidades Abertas para a Terceira Idade - e no atendimento ambulatorial da clientela adulta e idosa, vivenciada pelos integrantes do GENUTI.

Os temas desenvolvidos durante a intervenção educativa foram os seguintes: importância da alimentação para a saúde dos indivíduos; grupo de alimentos e suas funções no organismo; necessidades e recomendações nutricionais específicas para a população idosa; auto-cuidado em nutrição.

A distribuição temática foi de difícil precisão, tendo em vista a característica interativa

\footnotetext{
3 Grupo de Estudos de Nutrição na Terceira Idade - GENUTI, é um grupo de nutricionistas, existente desde 1993, com objetivo, de desenvolver atividades que visem melhorar a qualidade de vida da população da Terceira Idade.
} 
do curso, no qual o educador/nutricionista realizava adaptações constantes a fim de manter o conteúdo proposto e os interesses dos alunos.

A atividade de orientação específica consistiu em orientação alimentar individual, adaptada de um Guia Alimentar e impressa na forma de folheto. Este guia foi entregue no último dia de aula, juntamente com o resultado das avaliações do consumo alimentar e do estado nutricional. Essa atividade era realizada pela autora da pesquisa no encerramento das aulas de nutrição. Os alunos que participaram das aulas podiam, neste momento, esclarecer suas dúvidas quanto ao resultado de sua avaliação, e receber reforço da orientação geral. Em caso de situação que necessitasse prescrição dietética especial, foi recomendado procurar orientação profissional específica.

\section{RES U LTA D O S}

Os questionários utilizados para o pós-teste foram distribuídos aos alunos do segundo módulo, que haviam participado da primeira fase da pesquisa. Nessa época, o número de matriculados no segundo módulo era de 113 (58,0\% dos alunos que iniciaram o curso no módulo do semestre anterior). De todos os questionários distribuídos $(n=73), 44$ foram devolvidos e preenchidos corretamente; tal cifra corresponde a $60,3 \%$ do total. Houve uma perda de 22 questionários não devolvidos e 7 incompletos. Na Tabela 1 estão descritas as características desse grupo populacional, objeto das análises da pesquisa. A maioria foi constituída de indivíduos do sexo feminino e com idade inferior a 60 anos.

A comparação das variáveis dietéticas antes e depois da intervenção educativa relativa a esses 44 alunos (Tabela 2) pode-se observar uma diminuição significativa, do ponto de vista estatístico, referente ao colesterol e à quantidade total de lipídios, e da quantidade de proteínas.

Quanto ao teste relativo às noções conceituais sobre nutrição, observou-se um aumento da média de pontuação de 5,01 para 6,26, com diferença estatística significativa de 1,25 pontos (Tabela 3). Provavelmente, influenciaram esse aumento os temas educativos referentes à função das gorduras dietéticas e à substituição de alimento regulador.

Dentre os alunos investigados, dez informaram não ter realizado qualquer mudança na dieta nos últimos seis meses, por não considerar necessário. Na Tabela 4 estão listadas as alterações que os indivíduos referiram ter realizado. As principais modificações relacionaram-se ao tipo de alimento consumido, à ingestão de água e à maneira de preparar os alimentos.

Quanto aos motivos pelos quais os alunos alteraram suas dietas, verifica-se que $68,6 \%$ das citações indicam "por motivo de saúde" foi o principal deles. Além desse, as informações recebidas foram citadas por $24,0 \%$ como razão para efetuarem mudanças na dieta. Da mesma forma, houve referências a questões financeiras $(14,7 \%)$ e familiares $(14,7 \%)$, como fatores que justificaram as alterações dietéticas.

Tabela 1. Distribuição da população estudada após a intervenção educativa, segundo sexo e idade, São Paulo,1996/97.

\begin{tabular}{|c|c|c|c|c|c|c|}
\hline \multirow{3}{*}{ Grupo etário } & \multicolumn{4}{|c|}{ Sexo } & \multirow{2}{*}{\multicolumn{2}{|c|}{ Total }} \\
\hline & \multicolumn{2}{|c|}{ Masculino } & \multicolumn{2}{|c|}{ Feminino } & & \\
\hline & $n^{\circ}$ & $\%$ & $n^{\circ}$ & $\%$ & $n^{\circ}$ & $\%$ \\
\hline$<60$ anos & 3 & 12,5 & 21 & 87,5 & 24 & 54,5 \\
\hline$\geq 60$ anos & 0 & 0,0 & 20 & 100,0 & 20 & 45,5 \\
\hline Total & 3 & 7,0 & 41 & 93,0 & 44 & 100,0 \\
\hline
\end{tabular}


Tabela 2. Distribuição das médias referentes às variáveis dietéticas antes e depois da intervenção, São Paulo, $1996 / 97$.

\begin{tabular}{|c|c|c|c|c|c|}
\hline \multirow{2}{*}{ Variáveis dietéticas } & \multicolumn{2}{|c|}{ Antes } & \multicolumn{2}{|r|}{ Depois } & \multirow{2}{*}{$p$} \\
\hline & Média & DP & Média & DP & \\
\hline Energia (kcal) & $1.364,87$ & $\pm 404,6973$ & $1.251,04$ & $\pm 316,8282$ & 0,062 \\
\hline Proteínas (g) & 69,19 & $\pm \quad 17,4011$ & 61,50 & $\pm \quad 18,8747$ & 0,012 \\
\hline Carboidratos (g) & 174,26 & $\pm \quad 69,1167$ & 157,07 & $\pm \quad 46,1421$ & 0,092 \\
\hline Lipídios (g) & 48,55 & $\pm \quad 21,8461$ & 41,05 & $\pm \quad 14,6710$ & 0,004 \\
\hline Proteínas (\%) & 20,96 & 6,3844 & 20,51 & 4,4614 & 0,654 \\
\hline Carboidratos (\%) & 49,69 & 8,7972 & 51,03 & 8,8538 & 0,358 \\
\hline Lipídios (\%) & 29,38 & 6,6173 & 29,05 & 6,3182 & 0,757 \\
\hline Colesterol (mg) & 216,01 & $\pm 106,9127$ & 168,31 & $\pm \quad 88,0612$ & 0,004 \\
\hline Cálcio (mg) & 815,80 & $\pm 331,0591$ & 734,01 & $\pm 215,6706$ & 0,068 \\
\hline Ferro (mg) & 9,50 & 2,4912 & 9,21 & 3,0871 & 0,498 \\
\hline Vitamina $A(\mu E R)$ & 908,01 & $\pm 72,6366$ & 882,48 & $\pm 357,5583$ & 0,748 \\
\hline
\end{tabular}

Tabela 3. Médias e desvios-padrão (DP) das pontuações do conhecimento geral e específico, antes e depois da intervenção, São Paulo, 1996/97.

\begin{tabular}{|c|c|c|c|c|c|c|c|}
\hline \multirow{2}{*}{ Conhecimentos } & \multicolumn{3}{|c|}{ Antes } & \multicolumn{3}{|c|}{ Depois } & \multirow{2}{*}{$p$} \\
\hline & Média & & DP & Média & & DP & \\
\hline Geral & 5,0144 & \pm & 2,083 & 6,2553 & \pm & 2,629 & 0,002 \\
\hline \multicolumn{8}{|l|}{ Específico (Por temas) } \\
\hline 1. Alimentos ricos em colesterol & 0,6400 & \pm & 0,226 & 0,1337 & \pm & 0,269 & 0,142 \\
\hline 2. Alimentos que colaboram no controle da hipercolesterolemia & 0,1509 & \pm & 0,234 & 0,1809 & \pm & 0,259 & 0,544 \\
\hline 3. Características da alimentação saudável & 0,4419 & \pm & 0,453 & 0,5465 & \pm & 0,447 & 0,254 \\
\hline 4. Prevenção da osteoporose & 0,6628 & \pm & 0,446 & 0,6512 & \pm & 0,444 & 0,900 \\
\hline 5. Função das gorduras dietéticas & 0,0465 & \pm & 0,213 & 0,1860 & \pm & 0,394 & 0,013 \\
\hline 6. Alimentos energéticos & 0,2151 & \pm & 0,356 & 0,2674 & \pm & 0,395 & 0,399 \\
\hline 7. Formas de consumo dos alimentos & 0,4651 & \pm & 0,368 & 0,5116 & \pm & 0,401 & 0,511 \\
\hline 8. Prevenção do envelhecimento precoce & 0,5349 & \pm & 0,505 & 0,4767 & \pm & 0,499 & 0,528 \\
\hline 9. Mudanças das necessidades em função da idade & 0,3837 & \pm & 0,342 & 0,4420 & \pm & 0,381 & 0,490 \\
\hline 10. Substituição de alimento regulador & 0,2326 & \pm & 0,427 & 0,6628 & \pm & 0,472 & $<0,001$ \\
\hline 11. Função dos alimentos reguladores & 0,5581 & \pm & 0,502 & 0,6628 & \pm & 0,472 & 0,183 \\
\hline 12. Mudanças da dieta pelo envelhecimento & 0,6047 & \pm & 0,495 & 0,7326 & \pm & 0,441 & 0,147 \\
\hline 13. Fonte dos nutrientes antioxidantes & 0,6512 & \pm & 0,482 & 0,6047 & \pm & 0,495 & 0,570 \\
\hline
\end{tabular}

Tabela 4. Distribuição da população estudada após a intervenção educativa, segundo modificações dietéticas referidas, São Paulo, 1997.

\begin{tabular}{lll}
\hline Modificações dietéticas & $\mathrm{n}=4$ & $\%^{(*)}$ \\
\hline Tipos de alimentos consumidos & 22 & 50,0 \\
Ingestão de água & 15 & 34,1 \\
Maneira de preparar os alimentos & 14 & 31,8 \\
Quantidade ou horário de refeições & 17 & 38,6 \\
Quantidade de alimentos (em geral ou específicos) & 16 & 36,4 \\
Não realizaram modificações & 10 & 22,7 \\
\hline
\end{tabular}

*Porcentagem calculada em relação aos 44 participantes. 
Tabela 5. Distribuição da população estudada após a intervenção educativa, segundo as variáveis relativas aos recursos informativos utilizados, São Paulo, 1997.

\begin{tabular}{llll}
\hline Variáveis & Categorias & $n$ & $\%{ }^{\left({ }^{*}\right)}$ \\
\hline Fonte de Informação & UATI(*2) & 25 & 96,1 \\
& Médicos & 21 & 80,8 \\
& Nutricionistas & 20 & 76,9 \\
& TV & 6 & 23,1 \\
Recurso informativo mais importante & Revistas & 4 & 15,4 \\
& Jornais & 3 & 11,5 \\
& Médicos & 8 & 30,8 \\
& Todas as recebidas & 7 & 26,9 \\
& UATI + Nutricionistas & 7 & 26,9 \\
\hline
\end{tabular}

*1 Porcentagem calculada em relação aos 26 participantes que preferiram utilizar algum recurso informativo para as modificações dietéticas. *2 UATI - Universidade Aberta à Terceira Idade.

Quanto à fonte de informação que colaborou para que houvesse mudanças na dieta - independentemente do motivo de sua realização, os dados da Tabela 5 apontam como destaque a própria Universidade Aberta para a Terceira Idade, com 75,6\% das citações, seguida do médico com $63,4 \%$ e do nutricionista com $60,1 \%$. No caso, um dos nutricionistas citados não pertencia aos quadros de UATI. Pelo sistema de livre escolha, ao eleger qual a fonte de informação mais importante, verificou-se que as mais cotadas foram: o médico, com $27,6 \%$, e as citações "todas as assinaladas", registrando $24,0 \%$.

\section{I S C U S S Ã O}

O predomínio da população feminina nesta atividade educativa é decorrente da elevada participação de mulheres em Universidades Abertas para a Terceira Idade. Aparentemente, as mulheres tornam-se mais sensibilizadas às necessidades demandadas para a promoção de saúde, em decorrência de sua experiência na utilização dos serviços de saúde em outras fases da vida, em especial, para realização do pré-natal, parto e puericultura.

Importa destacar que a mudança do comportamento alimentar é um ponto funda- mental para avaliar a efetividade da educação nutricional ${ }^{6}$, os resultados encontrados nesta pesquisa apontam mudanças de vários aspectos do consumo e do comportamento alimentar entre os participantes da intervenção nutricional educativa avaliada.

As modificações das dietas identificadas na presente pesquisa apresentam características positivas, no que se refere à diminuição do consumo de lipídios e do colesterol. Vale ressaltar que, apesar dessa alteração na quantidade de lipídios, não houve modificação de modo significativo na participação energética dos macronutrientes e no total energético da dieta.

Dios-Sanz et al. ${ }^{17}$ obtiveram uma diminuição significativa no total energético, às custas da redução no consumo de gordura total e, especialmente, de gordura saturada, ao realizarem um programa educativo com o objetivo de reduzir os elementos de risco presentes na dieta.

Doshi et al. ${ }^{18}$, por sua vez, ao avaliarem uma intervenção educativa entre idosos negros, após dez semanas com orientação nutricional (duas vezes por semana), acompanhada de atividade física, não encontraram diferença entre o valor energético da dieta inicial e aquela posterior à intervenção. Essas alterações no consumo 
alimentar estão em consonância com os achados de Constants et al. ${ }^{19}$ que, ao desenvolverem intervenção educativa em UATI, na França, também obtiveram um resultado positivo. Esses autores encontraram um aumento estatisticamente significativo do consumo de cálcio, que era o objeto de estudo da intervenção realizada.

No caso da presente pesquisa, além das mudanças ocorridas no consumo alimentar, verifica-se o aumento na pontuação da avaliação das noções conceituais sobre nutrição de modo global e em alguns temas específicos. Intervenções educativas sem seguimento de apoio, tendem a alterar as práticas alimentares, no máximo, por seis meses ${ }^{6}$. Esses mesmos autores afirmam que as mudanças de conhecimento também podem regressar a valores inferiores à fase de pré-teste, após seis meses.

Nos seis meses pós-teste da presente pesquisa, todavia, não ocorreu este retorno, mas sim, uma melhora em relação à situação inicial. Algumas práticas citadas como tendo sido modificadas podem estar colaborando na melhoria da qualidade da alimentação como um todo. Por exemplo: alterações na mastigação, maior fracionamento das refeições e o aumento da ingestão de água.

A saúde foi o fator mais citado como determinante das alterações de comportamento realizada. Brombach ${ }^{20}$, em estudo qualitativo em que entrevistou 60 mulheres entre, 64 e 94 anos de idade, sobre os hábitos de beber e de comer encontrou dados semelhantes. Esse autor verificou que, o jeito de comer somente foi modificado pelo casamento ou em decorrência de implicações na saúde.

Os fatores econômicos e familiares, apesar de constituírem uma pequena porcentagem, reforçam a idéia de que a determinação dos hábitos alimentares está relacionada a diferentes aspectos. As informações e conhecimentos, contudo, podem ajudar na tomada de decisão, especialmente no que se refere à quantidade de alimentos ${ }^{6}$.
A decisão adequada, relativa à nutrição, pode estar associada à sua fonte de informação. Em geral, as mais comuns são: revistas, jornais, livros de receitas, televisão e as consultas médicas.

O médico teve um papel importante na determinação das modificações referidas em relação à quantidade de alimentos. Também verificou-se que o médico é a fonte de informação sobre nutrição mais citada em relação às mudanças de comportamento alimentar, em pesquisas com idosos.

Muitas evidências indicam que os profissionais da área de saúde, para estarem habilitados na orientação sobre dieta e nutrição, necessitam de treinamento e formação adicional. Pesquisa realizada com médicos e enfermeiras concluiu que esses profissionais reconhecem o nutricionista como o integrante da equipe de saúde mais habilitado para desenvolver orientação alimentar ${ }^{22}$. Estes dados corroboram os encontrados por Boog7, em investigação sobre o mesmo tema junto à equipe de saúde de serviços públicos de saúde, em cidade do interior do estado de São Paulo na qual a finalidade era discutir a implementação de atividades de educação nutricional, por intermédio de médicos e enfermeiras, que atuavam na linha de frente de serviços públicos de saúde. Nesta se conclui que o nutricionista é o profissional habilitado, por formação, para desenvolver programas e ações de educação nutricional - fato que é reconhecido pelas equipes e pelos usuários dos serviços.

Quanto à importância da ação educativa, verificou-se que esta teve um papel importante na decisão das mudanças referidas. As atividades educativas da UATI foram referidas, pela maioria dos alunos, como fonte de informação sobre nutrição. Os freqüentadores desses projetos sentem-se gratificados pela oportunidade de acesso à informação, e reconhecem sua validade para a sua saúde e qualidade de vida ${ }^{14}$. As UATI são excelentes espaços para a promoção de saúde, confirmando, assim, o papel destas agências como fontes de informações para a população usuária ${ }^{19}$. 


\section{O N C L U S Ã O}

As atividades desenvolvidas na Universidade Aberta para a Terceira Idade devem atender às necessidades e expectativas da população que procura estes projetos, ou seja, devem adequar o conteúdo das atividades às características dessa população, motivando-a para a participação cada vez mais efetiva no processo de auto-cuidado.

As Universidades Abertas à Terceira Idade, de muitas formas, estão mantendo as pessoas ativas e saudáveis, contribuindo, assim, para sua maior autonomia, seu direito ao trabalho, ao lazer, à informação e à educação. É uma estratégia importante para preparar a sociedade para uma realidade cada vez mais emergente: o aumento da população idosa no mundo, em especial nos países subdesenvolvidos.

Apesar das dificuldades metodológicas inerentes a este tipo de estudo, e mesmo que não se possa inferir que as modificações verificadas tenham ocorrido estritamente por conta das atividades educacionais, os resultados positivos desta pesquisa indicaram a tendência às modificações nas práticas alimentares e nas noções conceituais sobre nutrição, em decorrência da intervenção educativa.

\section{REFER Ê NCIAS}

1. Papaléo Netto M, Ponte JR. Gerontologia: a velhice e o envelhecimento em visão globalizada. São Paulo: Atheneu; 2002. p.3-12.

2. World Health Organization. Health of elderly: background document. Geneva; 1995. (WHO/HPR/ HEE/95.1).

3. Organización Mundial de la Salud. Dieta, nutrición y prevención de enfermedades no-transmisibles. Ginebra; 1990. (OMS - Serie de Informes Técnicos, 797).

4. Sahyoun NR. Nutrition education for the healthy elderly population: Isn't it time? J Nutr Educ Behav. 2002; 34 Suppl 1:\$42-7.
5. Aranceta B J. Educación nutricional. In: Serra Majem L1, Aranceta Bartrina J, Mataix Verdú J. Nutrición y salud pública: métodos, bases científicas y aplicaciones. Barcelona: Masson; 1995. p.66-72.

6. Contento I, Balch GI, Bronner YL, Paige DM, Gross $S M$, Bisignani $L$, et $a l$. The effectiveness of nutrition education and implications for nutrition policy, programs and research: a review of research. J Nutr Educ. 1995; 27(6):285-415.

7. Boog MCF. Educação nutricional em serviços públicos de saúde: em busca de espaço para ação efetiva [doutorado]. São Paulo: Faculdade de Saúde Pública, Universidade de São Paulo; 1996.

8. Coppard L. La autoatención de la salud y los ancianos. In: Organización Panamericana de la Salud. Hacia el bienestar de los ancianos. Washington DC; 1985. (OPS-Publicación científica, 492).

9. Quintero MO. El autocuidado. In: Anzola Pérez E, Galinsky D, Morales Martinez F, Salas AR, Sánchez Ayéndez M. La atención de los ancianos: un desafio para los años noventa. Washington, DC: OPS; 1994. (OPS - Publicación Científica, 546).

10. Abusabha R, Hsieh KH, Achterberg C. Dietary fat reduction strategies used by a group of adults aged 50 years and older. J Am Diet Assoc. 2001;101(9):1024-30.

11. Jordão Netto A, Saadi F. Breve avaliação do projeto Universidade aberta para a terceira idade da PUC-SP. São Paulo; 1995.

12. Scala MA. Going back to school: participation motives and experiences of older adults in an undergraduate classroom. Educ Gerontol. 1996; 22:747-73.

13. Telaroli Jr R, Machado JCMS, Carvalho F. Educação em saúde na terceira idade: avaliação de um programa de extensão universitária. Gerontologia. 1997; 5(2):55-65.

14. Assis M, Pacheco LC, Menezes IS. Repercussões de uma experiência de promoção da saúde no envelhecimento: análise preliminar a partir das percepções dos idosos. [periódico online] Textos 
Envelhecimento 2002; 5(7). Disponível em <URL: http://www.unati.uerj.br. [2003, set 20].

15. Universidade Federal de São Paulo. Centro de Informação em Saúde. Sistema de Apoio à decisão em nutrição [programa de computador] Versão 2.5. São Paulo: UNIFESP; 1993.

16. Dean AG, Dean JÁ, Burton AH, Dicker RC. Epi Info, Version 5: a word processing database and statistics program for epidemiology on micro-computers. Centers for Disease Control. Atlanta, Georgia, USA, 1990.

17. Dios Sanz JJ, Fernández Estívariz C, Olmo Garcia D, Alcázar Lázaro V, Gargallo Fernández M, Miranda Nicolás I, et al. Impacto de un programa educativo nutricional sobre el perfil lipídico y los hábitos alimentarios de una población adulta laboralmente activa. Nutr Hosp. 1996; 11(6):334-8.

18. Doshi NJ, Hurley RS, Garrison MF, Stombaugh IS, Rebovich EJ, Wodarski LA, et al. Effectiveness of nutrition education and physical fitness training program in owering lipid in the black elderly. J Nutr Elder. 1994; 13(3):23-33.

19. Constants T, Delarue J, Rivol M, Theret V, Lamisse F. Effects of nutrition education on calcium intake in the elderly. J Am Diet Assoc. 1994; 94(4):447-8.

20. Brombach C. The EVA-study: Meal patterns of women over 65 years. J Nutr Health Aging. 2001; 5(4):263-5.

21. Charlton KE. The nutrient intake of elderly men living alone and their attitudes towards nutrition educacion. J Hum Nutr Diet. 1997; 10:343-52.

22. Macario E, Emmons K, Sorensen G, Hunt M, Rudd $M$. Factors influencing nutrition education for patient with low literary skills. J Am Diet Assoc. 1998; 98(5):559-64.

Recebido para publicação em 6 de fevereiro de 2003 e aceito em 29 de junho de 2004. 